Professionals (HCPs) are asked about CBPs, let alone where they would direct patients to if they could not answer such queries themselves. This study describes the nature of patient requests for CBPs as well as HCPs' knowledge of CBPs and confidence in responding to such enquiries in a large teaching hospital.

Methods A bespoke 16-item survey was designed using a combination of multiple response and free text questions. The final version was reviewed by a Palliative Care Consultant and an Advanced Clinical Pharmacist. The instrument was distributed to doctors, nurses, pharmacists and allied professionals working in Oncology and Haematology in a Regional Cancer Centre over six weeks in Spring 2019. All data were anonymised. Responses were compiled and analysed using Microsoft Excel.

Results 114 completed questionnaires were returned. 49\% of respondents were asked about CBPs at least monthly. Most enquiries were about both disease treatment and symptom control, especially pain (85\%) and nausea (49\%). $77 \%$ of HCPs agreed that it was at least somewhat a part of their role, but only $5 \%$ felt confident talking about cannabis; $94 \%$ agreed they need more support or guidance on the topic. Concerningly, $22 \%$ of all respondents, including 38\% of doctors, would direct a patient to the internet for further information on medicinal cannabis.

Conclusion HCPs are asked frequently about CBPs yet they profoundly lack both knowledge and confidence when it comes to dealing with enquiries. Accordingly many patients end up being directed to the internet by HCPs for further information. There is a clear need for compulsory education and responses suggest staff would be happy to engage.

\section{A QUALITATIVE STUDY OF NURSING ATTITUDES TOWARDS ADMINISTERING ANTICIPATORY MEDICATION FOR SYMPTOM CONTROL TO DYING PATIENTS IN A HOSPITAL SETTING}

Fatima Shah, Fiona Dakin, Christine Hirsch, John Speakman, Jon Tomas. University of Birmingham, University Hospital Birmingham NHS Trust

\subsection{6/spcare-2020-PCC.104}

Background Anticipatory medications are regularly prescribed for subcutaneous administration to patients in their last weeks or days of life in order to relieve symptoms. Once prescribed, nurses are required to use their own judgment to assess the patient's need for medication. Although there is some literature reporting nursing experience in administration of anticipatory medicines in the community, information from the acute hospital setting is less well documented. We sought to explore awareness, knowledge and confidence of hospital nurses when administering as required (PRN) medicines for symptom control to dying patients.

Methods Semi-structured interviews were undertaken with qualified nurses. Purposive sampling was used targeting wards with higher death rates. An Interview guide was developed and piloted with the specialist Supportive and Palliative Care nursing team. Ethical approval was obtained. Interviews were audio-recorded, transcribed anonymously, coded and analysed using thematic analysis.

Results Ten interviews were conducted. Interviewees had been qualified between 1 and 10 years. Three main themes emerged from the interviews: education, nursing experience, and factors influencing nursing decision to administer PRN drugs. Newly qualified staff were less confident in administering PRN drugs, checking doses or questioning prescribing. Factors such as workload and family presence were recognised as influencing decisions to administer PRN drugs. Training, although provided regularly in the Trust, was considered difficult to access by the interviewees.

Conclusion In this large teaching hospital with a Supportive and Palliative Care team and an electronic prescribing system, although nurses interviewed were generally confident about administering PRN medication at end of life, this was dependent on their nursing experience. Most of the interviewees were able to identify the Supportive and Palliative Care team as their first point of call for advice. Further discussion with the specialist team following publication of the final report will determine how to address the identified issues.

\section{AN AUDIT OF CONTINUOUS SUBCUTANEOUS SYRINGE PUMP DOSES IN ADULTS RECOGNISED AS DYING IN A DISTRICT GENERAL HOSPITAL}

Clare Smith, Susan Dargan. Ashford and St Peter's NHS Trust

\subsection{6/spcare-2020-PCC.105}

Introduction Symptom assessment and management is one of the key areas in NICE guidance (NG31). The Gosport enquiry shone a spotlight on the use of continuous subcutaneous syringe pumps (CSCP) inparticular proportionate and appropriate use of opioids and other medications at the End of Life (EOL). In response to this report we conducted an audit of the use of CSCPs in our District General Hospital (DGH).

The audit had 2 aims;

- (1)To understand the frequency of use of CSCPs in adults recognised as dying,

- (2) To determine the average doses of medications in the last CSCP prescription prior to death.

Methods This was a retrospective audit. Data was collected alongside data collection for the National Audit of EOL Care 2019. A data collection tool was designed by the specialist palliative care team (SPCT) and results analysed in Excel. Thirty-five notes and drug charts of patients who died in April and May 2019 were reviewed.

Results 35 case notes were reviewed. The median age of patients recognised as dying was 83 years old (min 64, $\max$ 95). The 3 most common diagnoses were; cancer (24\%), dementia (18\%), respiratory disease (18\%). Thirteen patients $(37 \%)$ had a CSCP prescribed. Of these $12 /$ $13(92 \%)$ contained an opioid; 6(50\%) Morphine, 4 (33\%) Alfentanil, and 2 (17\%) Oxycodone. The mean subcutaneous morphine equivalent dose was $10.7 \mathrm{mg}$ over 24 hours. Ten $(77 \%)$ contained medication for agitation, the majority 9/10 (90\%) contained Midazolam, with a mean 24 hours dose of $6.7 \mathrm{mg}$, and $6 / 13$ (46\%) contained Glycopyrronium.

Conclusion This audit shows that less than half of patients dying in hospital require a CSCP, and that average 24 hour doses were relatively low. The next step is to scrutinize the notes and symptom observations charts to determine whether objectively symptoms at the EOL were well controlled with these doses. 